\title{
The Profile of Hepatic Gene Expression of Glucose Metabolism in Mice on High Fat Diet
} Rihab El-Fadel Mohamed ${ }^{1}$,

\section{Supervised by: Dr. Nasser Rizk ${ }^{1}$, Dr. Abdelrahman ElGamal2 ${ }^{2}$ Ms. Ameena Fadel ${ }^{3}$, Ms. Dina Elsayegh ${ }^{4}$ ${ }^{1}$ Biomedical Science Department, College of Health Sciences, Qatar University, Doha, Qatar ${ }^{2}$ Biomedical Research center}

\begin{abstract}
Obesity is a growing problem worldwide, and recent data indicated that $20 \%$ of the populations would be obese. Obesity arises as a multifactorial disease caused by inherited traits that interact with lifestyle factors such as diet and physical activity. The liver plays an essential role in the gluco-regulation via regulating glucose, lipid and protein metabolism. The process of glucose metabolism is controlled by a range of molecular mechanisms and genes which affect the metabolism of the liver during intake of high fat diet (HFD). The objective of this research is to investigate the profile of hepatic gene expression of glucose metabolism in mice on HFD treated with leptin ( $5 \mathrm{mg} / \mathrm{kg}$ BW Ip injection). Ten wild type CD1 mice fed on HFD is used for this study, where groups are control (vehicle - leptin) and test group (vehicle + leptin). Body weight (BW) was measured, and blood chemistry, insulin and leptin were measured at the end of the experiments. Total RNA was isolated from the liver tissue, and RTPCR profiler array technology was used to evaluate the mRNA expression of 84 essential genes of hepatic glucose metabolism. The data of the BW and blood chemistry are not significantly different between the two groups. Leptin treatment enhanced the metabolic pathways and the candidate genes of the different metabolic pathway; glycogen metabolism such as Gys1, Gys2 and Pygm, pentose phosphate shunt such as Rpia and suppressed the glycolysis such as Aldob, and TCA cycle such as Mdh1b. In conclusion, this study has shown that leptin could affect the profile of the hepatic mouse genes of glucose metabolism in the early stages of HFD to induce obesity.
\end{abstract}

\section{INTRODUCTION}

Obesity arises as a multifactorial disease caused by inherited traits that interact with lifestyle factors such as diet and physical activity. - Obesity in Qatar is alarming and it is the leading country in GCC countries with obesity prevalence reached up to $41.4 \%$ (39.5\% males, $43.2 \%$ females) (WHO, 2017).

In experimental animal models, Diet-induced obesity (DIO) in mice is similar to human obesity caused by lifestyle factors. In the liver, hepatic glucose production is upregulated with insulin resistance conditions and contributes to the hyperglycaemic effects and several critical molecules involved in this phenotyping(Kim and Ahn 2004).

The metabolism is controlled by a range of molecular mechanisms and genes which affect the metabolism of the liver during intake of high fat diet (HFD) to induce DIO.

Despite the extensive efforts done in previous studies, the genes identified thus far do not alone account for all of the variability in glucose homeostasis during HFD and DIO. Further insight may be obtained by conducting gene microarray studies during an early time of diet-induced obesity (DIO) and treatment by leptin as an anti-obesity hormone.

The study aims is to investigate the hepatic gene expression of glucose metabolism in mice on HFD and the effect of leptin treatment as an anti-obesity tool to counteract such early changes in the metabolism of glucose.

\section{RESULTS}

Table 1: The age, body weight, and biochemical parameters of CD1mice treaded with Vehicle+ saline and Vehicle+leptin

\begin{tabular}{|c|c|c|c|}
\hline & $\begin{array}{l}\text { Group 1 } \\
\text { (V+saline) } \\
N=5\end{array}$ & $\begin{array}{l}\text { Group } 2 \\
(\mathrm{~V}+\mathrm{lep}) \\
\mathrm{N}=5\end{array}$ & P value \\
\hline Age (weeks) & 10.0 & 10.0 & \\
\hline Body weight (mg) & $33.43 \pm 1.21$ & $34.11 \pm 2.22$ & 0.754 \\
\hline Insulin (ng/ml) & $3.16 \pm 1.31$ & $6.83 \pm 3.79$ & 0.437 \\
\hline Leptin (ng/ ml) & $1.32 \pm 0.12$ & $20.28 \pm 2.15$ & $<0.0001$ \\
\hline Glucose (mg/dl) & $157.44 \pm 6.07$ & $154.167 \pm 7.43$ & 0.925 \\
\hline $\begin{array}{l}\text { Triglycerides } \\
\text { (mg/dl) }\end{array}$ & $194.5 \pm 8.31$ & $182.9 \pm 5.48$ & 0.266 \\
\hline $\begin{array}{l}\text { Total Cholesterol } \\
(\mathrm{mg} / \mathrm{dl})\end{array}$ & $192.9 \pm 20.51$ & $154.6 \pm 3.48$ & 0.076 \\
\hline ALT (U/L) & $24.23 \pm 4.31$ & $21.42 \pm 2.77$ & 0.586 \\
\hline
\end{tabular}

Data represented as mean and standard error of mean (SEM). Results were analyzed using the Student's $t$ test. Two tailed $p$ values are significant $\leq 0.05$
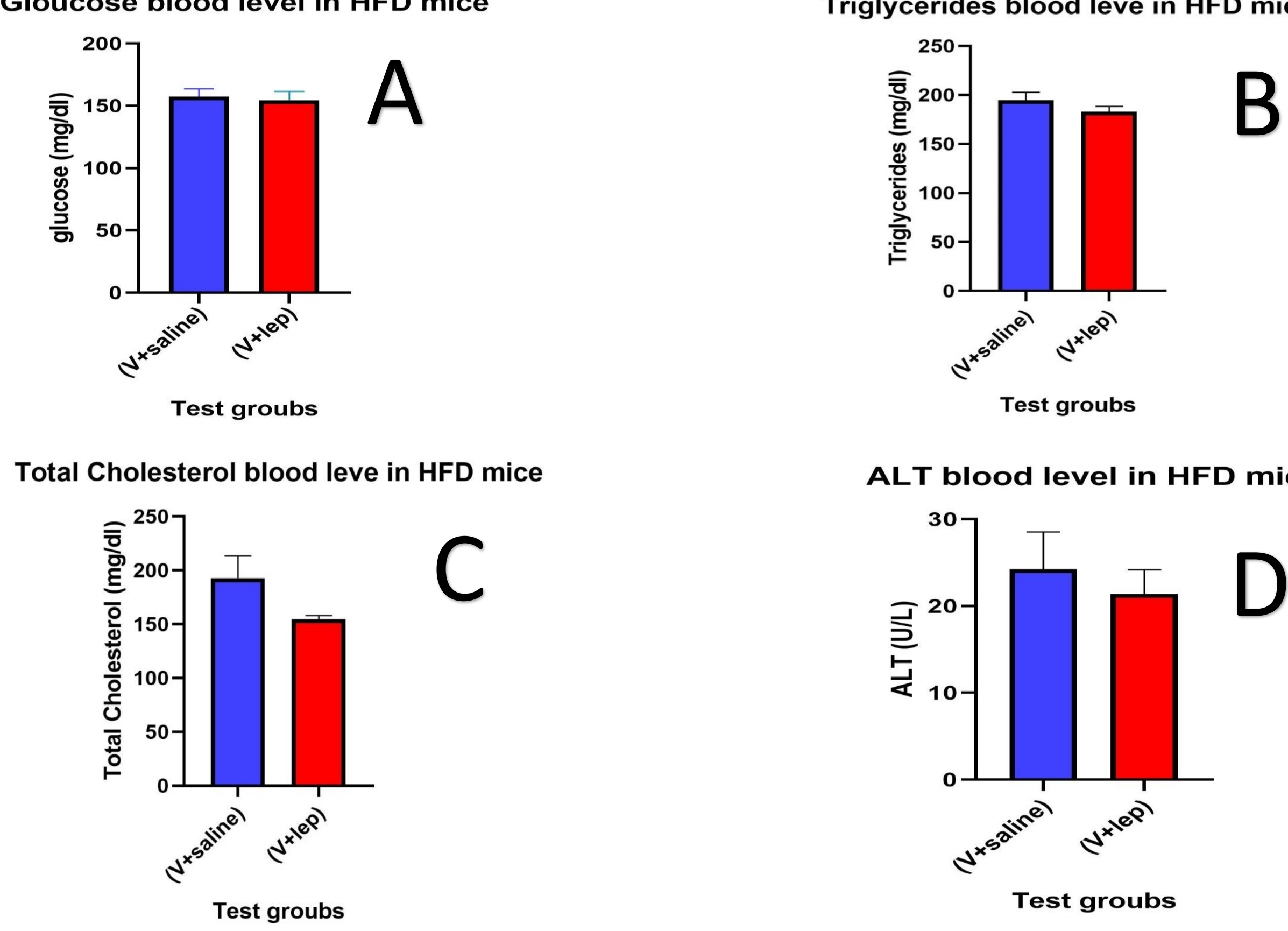

B

Figure A, B, C, and D represents the mean and SEM of biochemical tests in control group and group treated with leptin.
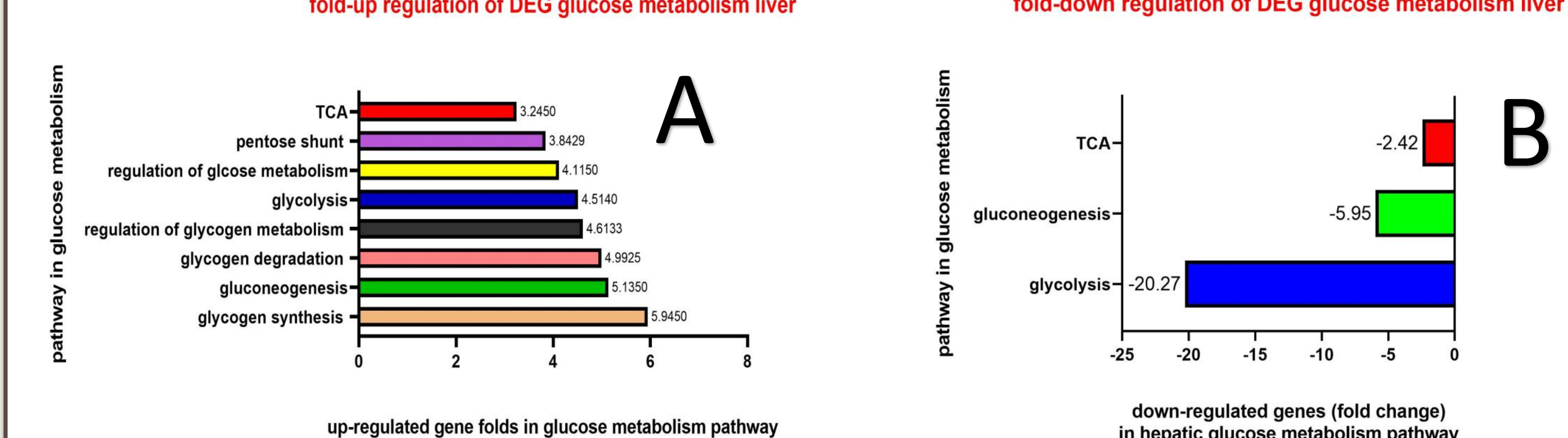

Figure A \&B shows the fold changes in pathways of different glucose metabolism of the up and down regulated gene after the leptin treatment compared to the control group.

\section{METHODOLOGY}

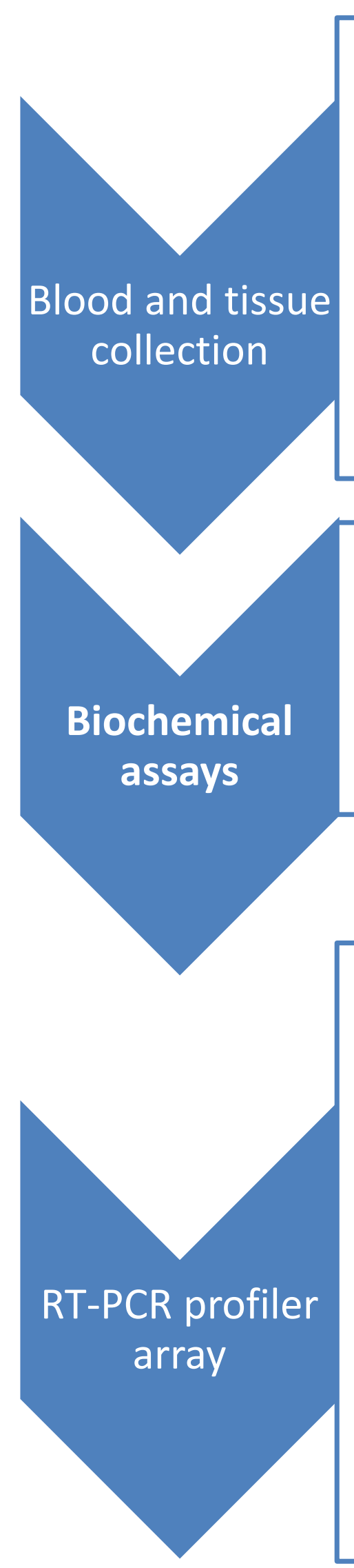
liquid nitrogen. $20^{\circ} \mathrm{C}$ until use. lab mice fed on HFD is used for this study, where groups are assigned based on the leptin treatment with and without treatment

- Food intake and body weight of the mice is measured at 1,3, 6,12 and $24 \mathrm{hr}$. post leptin injections. At the end of the treatment, liver tissues was collected quickly and snap frozen in

blood samples collected, and plasma separated.

- Leptin, and insulin, levels were determined with ELISA kits as based on manufacturer' protocol (Crystal.Chem, USA).

Fasting glucose, triglycerides, total cholesterol, and ALT were measured by photometric assays, based on the amateur's procedure provided by fortress.

- Total RNA was extracted from the liver tissues by the miRNeasy (Qiagen), and Qubit checked its quantity and qualityby using by using $\mathrm{EPOCH} 2$ microplate reader spectrophotometer to measure the absorbance at 260 and $280 \mathrm{~nm}$.

cDNA was generated from total RNA using High Capacity RNA-to-DNA Kit, The procedure was performed according to the manufacturer instructions and the final sample was stored at -

The expression level of genes involved in glucose metabolism from the liver, were analyzed the RT-PCR profile array using cyber green technology. Pathway analysis and gene ontology was use for analysis

\section{CONCLUSION}

The data of the BW and blood chemistry are not significantly different between the two groups.

treatment affects the regulation of multiple genes that are

involved in multiple metabolic pathways.

In glycogen synthesis pathway leptin up-regulates Gys1and Gys2 genes which enhances the process.

However, it enhanced the expression of Pygm and Pgm1genes which are involved in glycogen degradation. In the process of glycolysis, it does up-regulate the expression of Eno2 and Eno3 genes, and down-regulated the expression of Aldob gene, both effects enhanced the metabolism.

In the TCA cycle the expression of Mdh1b and Aldoc genes has increased, but the expression of Mdh1has decreased by the action of leptin. Fbp2 and G6pc3 which are involved in the process of gluconeogenesis their gene expression has increased, but Fbp1 and Pck1 decreased.

Genes involved in Pentose shunt metabolic pathway have been up-regulated, which are Rpia and Prps2.

Leptin treatment has increased the expression of genes involve in the regulation of glycogen metabolism which is Gsk3a and Phkg1, and genes involved in glucose metabolism regulation that is Pdk3 and Pdk4.

All of these change of regulation by leptin has shown a marked improvement in the hepatic glucose metabolism in HFD mice. This study has shown that leptin treatment is enhancing the metabolism by regulating the essential genes involved in differen hepatic glucose metabolism pathways such as glycogen metabolism, gluconeogenesis and glycolysis.

Leptin could be a treatment to obesity since it increases the metabolism and lowers the weight.

\section{REFERENCES}

作 glucokinase can be activated

WHO. (2017). Oatar Stepmise report 2012: chronic disease risk factor surveillance. Doha: Supreme Council of Health; 2015

http://www.sch.gov.qa/publications/publications, accessed 24 December 2015) Guo, Y., Darshi, M., Ma, Y, Perkins, G. A., Shen, Z., Haushalter, K. J, ... Taylor, S. S.
(2013). Quantitative proteomic and functional analysis of liver mitochondria from h fat diet (HFD) diabetic mice. Mol Cell Proteomics, 12(12), 3744-3758. doi:10.1074/mcp.M113.02744

Gao, M., Ma, Y., \& Liu, D. (2015). High-fat diet-induced adiposity, adipose O(3), e0119784-e0119784. doi:10.1371/journal pone 0119784

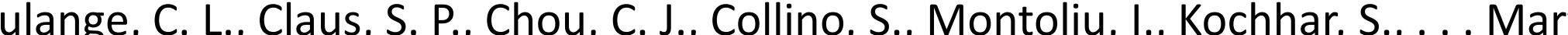
F.P. (2013). Early metabolic adaptation in C57BL/6 mice resistant to high fat diet induced weight gain involves an activation of mitochondrial oxidative pathways. $J$ Proteome Res, 12(4), 1956-1968. doi:10.1021/pr400051s

\section{ACKNOWLEGEMENTS}

Thanks to Dr. Nasser Rizk for his supervision, help, and continuous support. Thanks to those who are behind the success of this research: Dr. Abdelrahman ElGamal, Miss. Dina, Miss. Amina Saleh, Miss. Amina Aldous, Miss. Ovelia, and Miss. Aisha.

Lastly, we would like to thank the biomedical research center (BRC), and the biomedical department labs in the science building for allowing us to access their labs to work on our research. 